\title{
Leptophilic bosons and muon g-2 at lepton colliders
}

\author{
Eung Jin Chun and Tanmoy Mondal \\ School of Physics, Korea Institute for Advanced Study, \\ Seoul 02455, Republic of Korea \\ E-mail: ejchun@kias.re.kr, tanmoy@kias.re.kr
}

ABSTRACT: A light leptophilic boson (scalar or pseudoscalar) has been postulated to explain the muon g-2 anomaly and could be a portal to dark matter. Realizing the leptophilic nature of a singlet boson in the framework of the two-Higgs-doublet-Model of type-X, we identify the parameter space viable for the explanation of the updated muon g-2 discrepancy. It is then shown that such a hypothetical particle will be unambiguously ruled out or discovered via the Yukawa process at a lepton collider designed as a Higgs factory.

Keywords: Phenomenological Models

ArXiv EPrint: 2104.03701 


\section{Contents}

1 Introduction 1

2 A framework for leptophilic scalar and pseudoscalar 2

2.1 Scalar extension of $2 \mathrm{HDM} \quad 3$

2.2 Pseudoscalar extension of the 2HDM 4

2.3 Fit to the muon $g-2$ and lepton universality constraints 4

3 Search at Higgs factory $\quad \mathbf{5}$

3.1 Event simulation and signal selection 5

$\begin{array}{ll}3.2 & \text { Signal and background discrimination }\end{array}$

4 Results and discussion $\quad 6$

$\begin{array}{llr}5 & \text { Conclusion } & 8\end{array}$

\section{Introduction}

There have been extensive studies on new physics below the weak scale which can be looked for at various high-energy and high-intensity experiments in vast ranges of new particle masses and couplings [1]. One of the interesting possibilities is the presence of a light spin-0 particle mixing with the Higgs boson [2-5] which could be a portal to dark matter, or an inflaton. A scalar particle mixing with the SM Higgs boson interacts with the fermions with a coupling strength proportional to the fermion mass. Such a particle is severely constrained due to its hadronic interactions. On the other hand, if the scalar mediator couples predominantly to the leptons [6], substantial parameter space still remains open and viable to accommodate the $(g-2)_{\mu}$ anomaly which is confirmed by FNAL [7-9]. In connection to the dark sector, non-observation of any signal in the direct detection experiments may indicate a leptophilic mediator for dark matter. If the mediator is a pseudoscalar, the direct detection of dark matter becomes out of question since its interaction with matter is velocity suppressed [10].

A two-Higgs-doublet Model (2HDM) can provide a natural framework to realize the leptophilic nature of a light scalar or pseudoscalar. Phenomenology of scalar or pseudoscalar extension of $2 \mathrm{HDM}$ has been studied mainly in the context of dark matter [11-24]. A typical LHC search for a light leptophilic boson relies on the decay of the SM Higgs boson to a pair of light particles which subsequently decay into leptons. This could be an important discovery channel if it is allowed kinetically and the corresponding coupling is sizable. More direct searches can be performed through the Yukawa production followed by leptonic decays. The new results from BABAR Collaboration [25] have excluded a leptophilic scalar between $0.1 \mathrm{MeV}$ to a few $\mathrm{GeV}$ in the coupling range which can explain the 
$(g-2)_{\mu}$ anomaly. Incorporating the one- and two-loop contributions properly, we will see that the leptophilic scalar mass window of a few to $10 \mathrm{GeV}$ is still open to accommodate the muon $(g-2)$ anomaly. The other region below the BABAR limit has been ruled out by the electron beam dump experiments $[26,27]$.

The situation for a leptophilic pseudoscalar is completely different. First of all, the minimal 2HDM of type-X can accommodate the muon $(g-2)$ anomaly for large $\tan \beta$ and a CP-odd Higgs boson above $10 \mathrm{GeV}$ [28-32]. ${ }^{1}$ Considering a light pseudoscalar mixing with a heavier CP-odd Higgs boson, the muon $(g-2)$ favored region is extended to lower mass which is however above the BABAR reach, and there is no direct search limit so far.

A light particle at the $\mathrm{GeV}$ scale is in the border zone between high-intensity and highenergy searches, and hence it is challenging to extend the search limits on either side. The purpose of this paper is to see how far in the lower mass range future collider experiments can reach and check whether the muon $(g-2)$ region of a scalar and pseudoscalar can be covered. At a lepton collider, a leptophilic boson can be produced via the Yukawa process [40-46] where a tau lepton radiates a boson, and then decays almost exclusively to a pair of leptons. If allowed kinematically, it leads to the conventional signal of four tau-lepton final states. It is possible to probe the four-tau final state and use collinear approximation to reconstruct the mass at the ILC [46]. However, for a lighter boson, such a search method is not feasible as the decay products will be too collimated to be distinguished as two separate tau-tagged jets.

In this paper, we propose to search for a muon in association with an opposite sign charged track as the signal for a light leptophilic boson. The charged track will be adjacent to the muon since both of them originate from the decay of a collimated pair of tau leptons coming from the boson. Precision measurement of the Higgs boson is the prime target for any future lepton collider which will run as a 'Higgs factory' with a centre-of-mass energy of $250 \mathrm{GeV}$. We will consider ILC as an example and show that the proposed signal efficiently probes a light $\phi$ in the mass and coupling range beyond the BABAR limit. Our signature will be a complementary exploration of any spin- 0 particle in the $\mathrm{GeV}$ scale mass range. It is worthwhile to emphasize that the signals at the lepton collider under the consideration arise solely from the Yukawa interaction of a boson with leptons, and thus are decisive to confirm or disprove the presence of a leptophilic boson.

\section{A framework for leptophilic scalar and pseudoscalar}

We will briefly discuss the scalar and pseudoscalar extension of the 2HDM scenario [47-49] as a framework for an extra leptophilic boson. The scalar potential of $2 \mathrm{HDM}$ is given by,

$$
\begin{aligned}
V_{2 \mathrm{HDM}}= & -m_{11}^{2} \Phi_{1}^{\dagger} \Phi_{1}-m_{22}^{2} \Phi_{2}^{\dagger} \Phi_{2}-m_{12}^{2}\left[\Phi_{1}^{\dagger} \Phi_{2}+\Phi_{2}^{\dagger} \Phi_{1}\right]+\frac{1}{2} \lambda_{1}\left(\Phi_{1}^{\dagger} \Phi_{1}\right)^{2}+\frac{1}{2} \lambda_{2}\left(\Phi_{2}^{\dagger} \Phi_{2}\right)^{2} \\
& +\lambda_{3}\left(\Phi_{1}^{\dagger} \Phi_{1}\right)\left(\Phi_{2}^{\dagger} \Phi_{2}\right)+\lambda_{4}\left(\Phi_{1}^{\dagger} \Phi_{2}\right)\left(\Phi_{2}^{\dagger} \Phi_{1}\right)+\frac{1}{2} \lambda_{5}\left\{\left(\Phi_{1}^{\dagger} \Phi_{2}\right)^{2}+\left(\Phi_{2}^{\dagger} \Phi_{1}\right)^{2}\right\}
\end{aligned}
$$

\footnotetext{
${ }^{1}$ For some recent developments along this direction see [33-38]. A comprehensive discussion about BSM explanation of $(g-2)_{\mu}$ in the light of FNAL result can be found in [39].
} 
The dimensionful coupling $m_{12}^{2}$ breaks the $\mathbb{Z}_{2}$ charge introduced to forbid the flavor changing neutral current interactions. Since we are interested in leptophilic extensions, we will consider the type-X model where $\Phi_{1}$ and the right-handed leptons are odd under the $\mathbb{Z}_{2}$ symmetry, whereas all other particles are even under the same symmetry. Consequently, the Yukawa Lagrangian is given by,

$$
-\mathcal{L}_{Y}=Y^{u} \overline{Q_{L}} \widetilde{\Phi}_{2} u_{R}+Y^{d} \overline{Q_{L}} \Phi_{2} d_{R}+Y^{e} \overline{l_{L}} \Phi_{1} e_{R}+\text { h.c. },
$$

where $\widetilde{\Phi}_{2}=i \sigma_{2} \Phi_{2}^{*}$. The scalar doublets can be parameterized as, $\Phi_{i}=$ $\left(\phi_{i}^{+},\left(v_{i}+\Phi_{i}^{0 R}+i \Phi_{i}^{0 I}\right) / \sqrt{2}\right)^{T}$ and as usual $\tan \beta=v_{2} / v_{1}$ and $v=\sqrt{v_{1}^{2}+v_{2}^{2}}=246 \mathrm{GeV}$.

\subsection{Scalar extension of $2 \mathrm{HDM}$}

In this scenario a $\mathrm{CP}$ even scalar $(S)$ is added to the scalar sector of $2 \mathrm{HDM}$. The scalar potential mixing $S$ with the doublets $\Phi_{1,2}$ is given by,

$$
V_{\mathrm{S}-\Phi}=A_{12}\left[\Phi_{1}^{\dagger} \Phi_{2}+\Phi_{2}^{\dagger} \Phi_{1}\right] S+A_{11} \Phi_{1}^{\dagger} \Phi_{1} S+A_{22} \Phi_{2}^{\dagger} \Phi_{2} S+\frac{\lambda_{1 S}}{2} \Phi_{1}^{\dagger} \Phi_{1} S^{2}+\frac{\lambda_{2 S}}{2} \Phi_{2}^{\dagger} \Phi_{2} S^{2}
$$

After the spontaneous electroweak symmetry breaking (EWSB) the scalar sector is comprised of on charged Higgs, one CP-odd neutral scalar and three CP-even neutral scalars. The rotation matrix which diagonalize the $3 \times 3$ neutral CP-even scalar mass matrix will mix the gauge eigenstates,

$$
\left(\begin{array}{c}
\Phi_{1}^{0 R} \\
\Phi_{2}^{0 R} \\
S
\end{array}\right) \approx\left(\begin{array}{ccc}
-s_{\alpha} & c_{\alpha} & s_{\theta_{1}} \\
c_{\alpha} & s_{\alpha} & s_{\theta_{2}} \\
-s_{\theta_{1}} & -s_{\theta_{2}} & 1
\end{array}\right)\left(\begin{array}{c}
h_{125} \\
H \\
\phi
\end{array}\right) .
$$

for small $\sin \theta_{1,2}$. Here $H$ is heavy CP-even scalar which we assume to be degenerate with the charged scalar $\left(H^{ \pm}\right)$and the pseudoscalar $(A)$ in our analysis. The mass eigenstates $\phi$ is supposed to be light to explain the muon $g-2$ anomaly.

After symmetry breaking we can write the interaction of the light scalar $\phi$ with the fermion mass eigenstates using eq. (2.1) and (2.3),

$$
\mathcal{L}_{\text {Yukawa }}^{\text {Physical }} \supset-\frac{m_{q}}{v} \frac{\sin \theta_{2}}{\sin \beta} \phi \bar{q} q-\frac{m_{\ell}}{v} \frac{\sin \theta_{1}}{\cos \beta} \phi \bar{\ell} \ell
$$

where $q=u, d$. From the above equation it is evident that $\phi$ will have a large coupling to leptons for large $\tan \beta$. The angle $\theta_{2}$ dictates the mixing of $\phi$ with $\Phi_{2}$ which is mostly the SM Higgs doublet near the alignment limit. By keeping this mixing small, it is possible to evade present bounds on light scalar coming from LHC and the proton beam dump experiments. The effective lepton Yukawa coupling of $\phi$ will be denoted by

$$
\xi_{\phi}^{\ell}=\frac{\sin \theta_{1}}{\cos \beta} \approx \tan \beta \sin \theta_{1}
$$

for large $\tan \beta$. 


\subsection{Pseudoscalar extension of the 2HDM}

In this case a $\mathrm{CP}$-odd scalar $P$ is added to the $2 \mathrm{HDM}$ sector. The additional scalar potential mixing $P$ with $\Phi$ reads as,

$$
V_{\mathrm{P}-\Phi}=i B_{12}\left[\Phi_{1}^{\dagger} \Phi_{2}-\Phi_{2}^{\dagger} \Phi_{1}\right] P+\frac{\lambda_{1 P}}{2} \Phi_{1}^{\dagger} \Phi_{1} P^{2}+\frac{\lambda_{2 P}}{2} \Phi_{2}^{\dagger} \Phi_{2} P^{2}
$$

After the EWSB the imaginary components of the doublets $\Phi_{i}^{0 I}$ mixes to form the Goldstone of the $Z$ boson and $\hat{A}\left(=-s_{\beta} \Phi_{1}^{0 I}+c_{\beta} \Phi_{2}^{0 I}\right)$. Then $\hat{A}$ mixes with $P$ to generate two mass eigenstates,

$$
\left(\begin{array}{l}
\hat{A} \\
P
\end{array}\right)=\left(\begin{array}{cc}
c_{\theta} & -s_{\theta} \\
s_{\theta} & c_{\theta}
\end{array}\right)\left(\begin{array}{l}
A \\
\phi
\end{array}\right)
$$

where the mixing angle can be written as,

$$
\tan 2 \theta=\frac{2 B_{12} v}{m_{A}^{2}-m_{\phi}^{2}}
$$

Note that we used the same notation $\phi$ for the scalar and pseudoscalar mass eigenstates. For our analysis, we will take the same mass for the heavy Higgs bosons of 2HDM: $A, H$ and $H^{ \pm}$. The light mass eigenstate $\phi$ will interact with fermions through the mixing as follows:

$$
\mathcal{L} \supset \frac{\sin \theta}{\tan \beta} \frac{m_{u}}{v} i \phi \bar{u} \gamma^{5} u+\frac{(-\sin \theta)}{\tan \beta} \frac{m_{d}}{v} i \phi \bar{d} \gamma^{5} d+\tan \beta \sin \theta \frac{m_{\ell}}{v} i \phi \bar{\ell} \gamma^{5} \ell .
$$

Again we define the effective lepton coupling as

$$
\xi_{\phi}^{\ell}=\tan \beta \sin \theta
$$

and the corresponding quark coupling becomes $\xi_{\phi}^{q} \approx \xi_{\phi}^{l} / \tan ^{2} \beta$ which is highly suppressed at large $\tan \beta$.

\subsection{Fit to the muon $g-2$ and lepton universality constraints}

Extending the analysis in [32] to the case of the scalar/pseudoscalar mixing with 2HDM, one can identify the parameter region of $\left(m_{\phi}, \xi_{\phi}^{l}\right)$ consistent with the new result on $a_{\mu}=$ $(g-2)_{\mu} / 2[8]:$

$$
a_{\mu}(\operatorname{Exp})-a_{\mu}(\mathrm{SM})=(251 \pm 59) \times 10^{-11} .
$$

Our $2 \sigma$ favored region does not change too much as there are a little shifts in both the central value and the error. We also plot the $2 \sigma$ bounds from the lepton universality conditions in the lepton (tau) decays and the leptonic $Z$ decays $(Z \rightarrow l l)$. For this, we take $\xi_{\phi}^{l}=\tan \beta \sin \theta \lesssim 100$, which corresponds to the tau coupling $\frac{m_{\tau}}{v} \tan \beta$ smaller than $\sim 1$. The heavy Higgs bosons are taken to be degenerate at $300 \mathrm{GeV}$ and thus their contribution to the muon $(g-2)$ is negligible. 


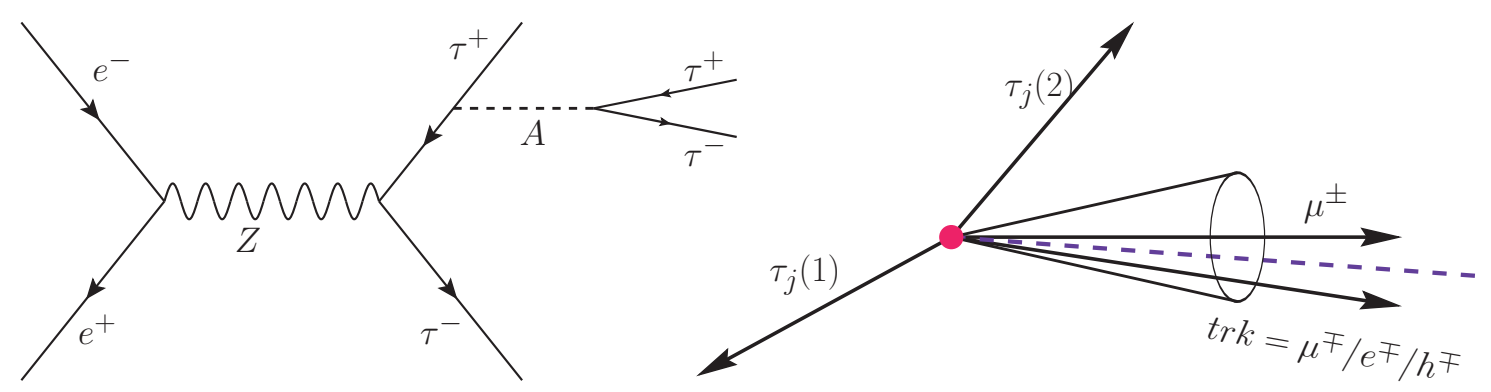

Figure 1. Signal topology of a light boson production at the lepton collider. The dashed line in the right panel depicts the direction of light boson $\phi$.

\section{Search at Higgs factory}

The Yukawa production of a leptophilic scalar or pseudoscalar is given by

$$
e^{+} e^{-} \rightarrow Z^{*} / \gamma^{*} \rightarrow \tau^{+} \tau^{-} \phi
$$

When $m_{\phi}>2 m_{\tau}$, which is the case under consideration, $\phi$ decays dominantly to a pair of taus. For a light $\phi$, its decay products will be collimated, and it is not feasible to isolate four final state tau leptons. To overcome this difficulty, we propose to look for a muon and a single charge track in the vicinity of the muon. In this case, one of the tau coming from $\phi$ will decay to a muon, and the other will give rise to a charged track. The charge track can originate from the leptonic decay mode or hadronic one-prong decay of the tau lepton, thus includes $85 \%$ of the tau decay products. In addition to the muon and track system, there will be two additional tau-tagged jets. The signal topology is shown in figure 1 . The dominant background comes from di-boson production,

$$
e^{+} e^{-} \rightarrow Z / \gamma^{*} Z / \gamma^{*} \rightarrow 2 \mu 2 \tau, 4 \tau \text {. }
$$

There is also a subdominant contribution from the Higgs-strahlung process.

\subsection{Event simulation and signal selection}

We use MadGraph5_aMC@NLO $[50,51]$ to generate parton level signal and background events and PYTHIA8 $[52,53]$ for the subsequent decay, showering and hadronization. For our analysis we have polarized beam with $P\left(e^{+}, e^{-}\right)=(+30 \%,-80 \%)$ [54]. The $\tau$ decays are incorporated via TAUOLA [55] integrated in MadGraph5_aMC@NLO. For detector effects we use Delphes3 [56] with the ILD detector card to simulate the detector effects. Jets are clustered using the longitudinal-kT algorithm $[57,58]$ with $R=0.4$. In Delphes3, we assumed the tagging efficiency of $\tau$ jets $\epsilon_{\tau}=90 \%$ [59]. Since we are interested a charge track close to the muon we remove the muon isolation criteria. We imposed the pre-selection criteria that all the tau tagged and the muon should have minimum energy of $20 \mathrm{GeV}$ and should have $|\eta|<2.3$ which corresponds to $|\cos \theta|<0.98$. In addition we require that both the tau-tagged jets and the muon should be well separated with $\Delta R \geq 0.4$.

There should be a single opposite sign charge track with $p_{T}>2.5 \mathrm{GeV}$ and the angular distance between the muon and the charge track $\Delta R\left(\mu^{ \pm}, \operatorname{trk}^{\mp}\right)$ should be less than 1.0. In 

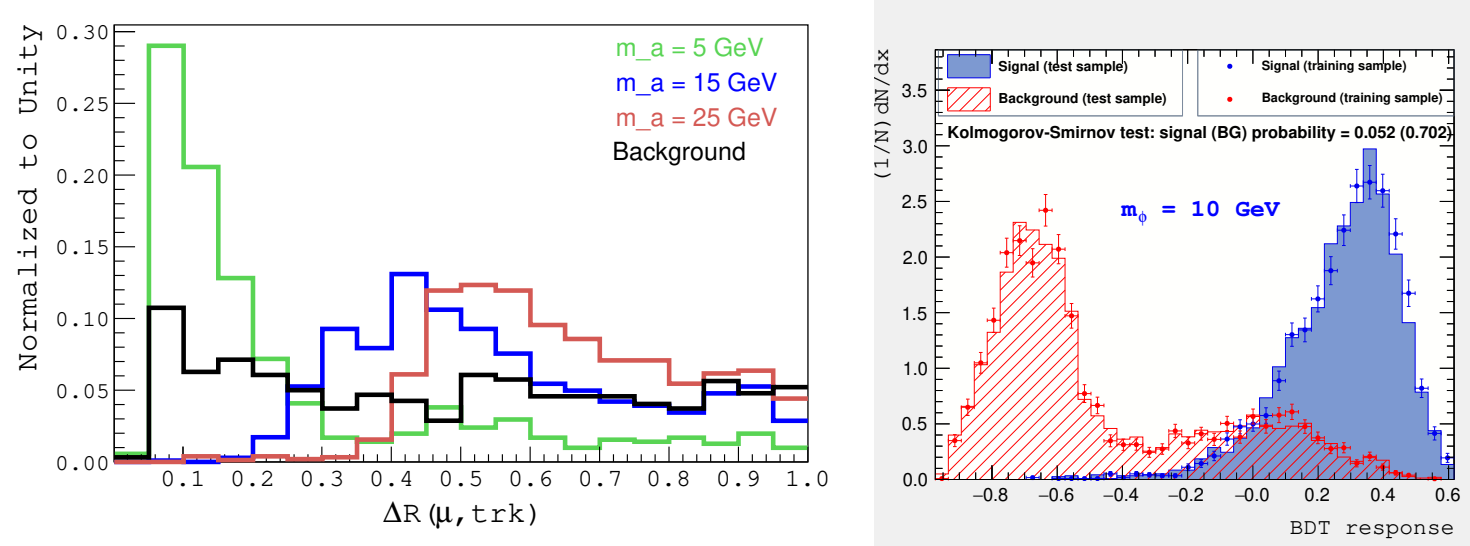

Figure 2. Angular separation between muon and a single charge track for different values of $m_{\phi}$. The black histogram shows the background distribution. Evidently the separation is small for light $\phi$ and increases as $m_{\phi}$ increases. Right Panel: here we show BDT response of signal and background for $m_{\phi}=10 \mathrm{GeV}$.

figure 2 we show the variation of $\Delta R\left(\mu^{ \pm}, \operatorname{trk}^{\mp}\right)$ for different $\phi$ mass and for background processed and as $m_{\phi}$ increases the angular separation increases.

\subsection{Signal and background discrimination}

We have used boosted decision tree (BDT) in the TMVA [60] framework to classify signal and background events. The following seven variable are used as input for the BDT: energy of muon and the two tagged jets, angular separation between the muon and the tau tagged jets and $\Delta R\left(\mu^{ \pm}, \operatorname{trk}^{\mp}\right)$. The BDT classifier is shown in right panel of figure 2 for $m_{\phi}=10 \mathrm{GeV}$. As expected $\Delta R\left(\mu^{ \pm}\right.$, trk $\left.^{\mp}\right)$ is the most important variable in the classifier followed by energy of muon.

\section{Results and discussion}

We analyze the signal significance with an integrated luminosity of $2000 \mathrm{fb}^{-1}$. We scan the mass of $\phi$ from $5 \mathrm{GeV}$ to $30 \mathrm{GeV}$ with step $2.5 \mathrm{GeV}$ and for each mass point we employed MVA classifier to obtain signal significance.

In figure 3 we have shown our main result in the $m_{\phi}-\xi_{\phi}^{l}$ parameter space. The effective lepton coupling $\xi_{\phi}^{l} \approx \sin \theta \tan \beta$ is explicitly shown eq. (2.5) for the scalar and in eq. (2.10) for the pseudoscalar. The blue (brown) patch in the left panel shows the parameter region which can explain the muon $(g-2)$ anomaly for a scalar (pseudoscalar) particle in the mass range from $10 \mathrm{MeV}$ to $100 \mathrm{GeV}$. The coupling required for the scalar boson via one loop is shown in the dotted blue lines. Since the contribution coming from the two-loop diagram becomes sizable and can cancel the one-loop contribution, a larger coupling is necessary when the scalar becomes heavy. The opposite behavior occurs for the pseudoscalar for which the two-loop contribution needs to dominate over the one-loop contribution. Hence, the pseudoscalar has to be heavier and larger coupling is required. The green and grey 

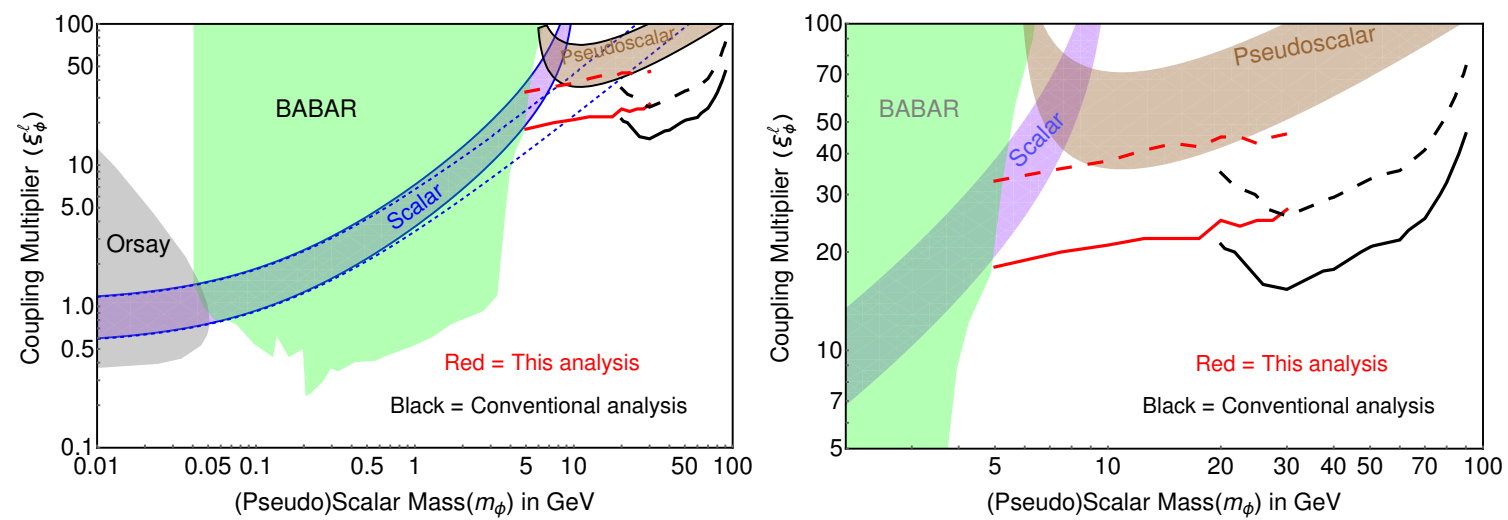

Figure 3. Parameter space of a leptophilic spin-0 particle explaining the muon $(g-2)$ anomaly. Blue (brown) colored region can explain $(g-2)_{\mu}$ when the spin-0 particle is a scalar (pseudoscalar). The negative two-loop effect in scalar case is apparent for higher mass range where total contribution in inside blue solid region differs from the blue dotted lines. Substantial part of the parameter space is constrained by the beam dump [27] (shown in gray) and BABAR [25] experiment (shown in green). Limit from ILC is shown in red and black curves where solid and dashed line corresponds to exclusion $(2 \sigma)$ and discovery $(5 \sigma)$ limit at ILC250 with $2 a b^{-1}$ of data. Limit of conventional analysis is taken from ref [46].

areas are excluded by BABAR and Orsay experiments. Evidently, $\phi$ has to be heavier than a few $\mathrm{GeV}$ to satisfy these constraints. Our results on the reach of ILC are shown in the black and red lines. The red solid and dashed curves display the $2 \sigma$ exclusion and $5 \sigma$ discovery limits at ILC with $2 a b^{-1}$ data. Since we have used BDT trained for each mass range the limit remains quite strong throughout. When $m_{\phi}$ is relatively heavy, the conventional search is possible. For completeness, we have shown the reach of such analysis in black solid (dashed) line which corresponds to $2 \sigma$ exclusion ( $5 \sigma$ discovery) region [46]. It is evident that ILC is capable of exploring all the still unconstrained viable region. In the right panel of figure 3 we have zoomed into the relevant parameter space.

The parameter space of an additional boson mixing with the 2HDM Higgs bosons gets constrained strongly by the lepton universality in the $Z$ and lepton $(\tau)$ decays [31, 32] as shown by the blue solid and dashed lines in figure 4 . The lepton universality constraint from the $\tau$ decay is sensitive to the value of $\tan \beta$ and we took $\tan \beta=85$ for the plot. Here, a large coupling multiplier is already constrained via the lepton universality, and the ILC will be able to explore the remaining parameter space without ambiguity. As before, the red and black solid (dashed) curves show exclusion (discovery) via charged track and conventional analysis respectively.

In leptophilic scenarios, like type-X $2 \mathrm{HDM}$, the coupling of the spin-0 particle with the leptons is proportional to the lepton masses, and the tau-Yukawa coupling provides collider signatures for such boson. The coupling of the quarks with the spin-0 particle is inversely proportional to $\tan \beta$ and are always small. In a more general approach, the aligned 2HDM considers couplings of leptons, up and down type quarks with the pseudoscalar is independent of each other. However, the quark couplings are severely restricted by neutral 


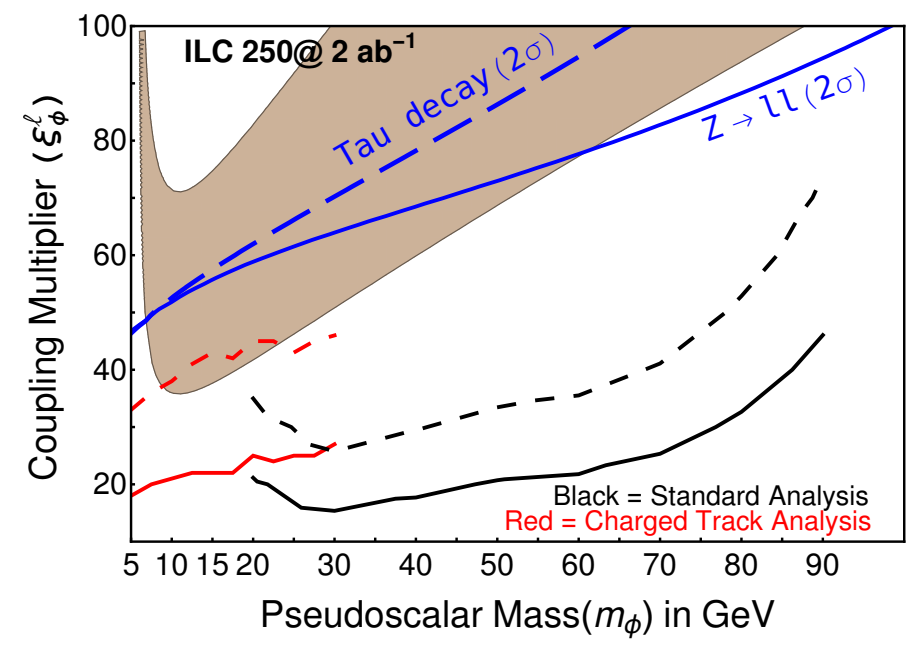

Figure 4. Parameter space in the pseudoscalar extended 2HDM model where the light additional pseudoscalar explain the $(g-2)_{\mu}$ anomaly. Strong constraints on the parameter space comes from the lepton universality in the $Z$ and $\tau$ decays. Although substantial parameter space is allowed from lepton universality, ILC will be able to explore the entire parameter space. Limit from ILC is shown in red and black curves where solid and dashed line corresponds to exclusion $(2 \sigma)$ and $\operatorname{discovery}(5 \sigma)$ limit at ILC250 with $2 a b^{-1}$ of data. Limit of conventional analysis is taken from ref [46].

meson mixing, $B_{s} \rightarrow \mu \mu$ and $b \rightarrow s \gamma$ observations [61-63], and the model relies on tauYukawa to provide the dominant contribution to $(g-2)_{\mu}$ via two-loop. Hence, a light pseudoscalar in aligned 2HDM will exhibit similar behaviour as in the type-X scenario, and one can simply recast our results for this case. Alternatively, it is possible to generate substantial $(g-2)_{\mu}$ at one loop with a $\mathcal{O}(100) \mathrm{GeV}$ scalar in muon specific [64] or general (type-III) 2HDM framework [65]. In muon specific case, the BSM scalars couples to muon and the collider signatures are muon rich. Similarly, in 2HDM-III, one has to rely on large flavour violating $\mu-\tau$ coupling, which can be probed indirectly via $\tau \rightarrow \mu \gamma, h_{125} \rightarrow \mu \tau$ decays. Since the mass scale of the new scalars are generally higher than the lepton collider 'Higgs factory', these models will show multi muon final state via Yukawa process at a high energy lepton collider like CLIC.

\section{Conclusion}

The existence of leptophilic spin-0 particles accommodated in the type-X $2 \mathrm{HDM}$ is well motivated, particularly, in view of the recent determination of the muon $(g-2)$ excess. Search for such a particle at the LHC relies on the exotic decay of the SM Higgs boson to these particles. On the other hand, BABAR and beam dump experiments have already excluded them in the sub-GeV mass range. The future lepton collider 'Higgs factory' will be an ideal place to directly explore $\mathrm{GeV}$ scale leptophilic bosons and their coupling to leptons. In a previous analysis, we showed that it is possible to explore four distinguished tau-lepton signature when the particle mass is few tens of $\mathrm{GeV}$. However, for the lighter mass range, this strategy will not work as the decay products of such particle start to merge. Here, we 
propose to probe a muon in association with a charged track as a possible signature for light leptophilic spin-0 particles. We have shown that the lepton collider will explore the entire $(g-2)_{\mu}$ allowed parameter space for both scalar and pseudoscalar particles.

\section{Acknowledgments}

TM would like to thank Akanksha Bhardwaj for useful discussions regarding BDT. TM (EJC) is supported by a KIAS Individual Grant PG073502 (PG012504) at Korea Institute for Advanced Study.

Open Access. This article is distributed under the terms of the Creative Commons Attribution License (CC-BY 4.0), which permits any use, distribution and reproduction in any medium, provided the original author(s) and source are credited.

\section{References}

[1] J. Beacham et al., Physics beyond colliders at CERN: beyond the Standard Model working group report, J. Phys. G 47 (2020) 010501 [arXiv: 1901.09966] [INSPIRE].

[2] Y.G. Kim, K.Y. Lee and S. Shin, Singlet fermionic dark matter, JHEP 05 (2008) 100 [arXiv:0803.2932] [INSPIRE].

[3] B. Batell, M. Pospelov and A. Ritz, Multi-lepton signatures of a hidden sector in rare $B$ decays, Phys. Rev. D 83 (2011) 054005 [arXiv:0911.4938] [InSPIRE].

[4] F. Bezrukov and D. Gorbunov, Light inflaton hunter's guide, JHEP 05 (2010) 010 [arXiv: 0912.0390] [INSPIRE].

[5] J.D. Clarke, R. Foot and R.R. Volkas, Phenomenology of a very light scalar (100 MeV $<m_{h}<10 \mathrm{GeV}$ ) mixing with the SM Higgs, JHEP 02 (2014) 123 [arXiv:1310.8042] [INSPIRE].

[6] B. Batell, N. Lange, D. McKeen, M. Pospelov and A. Ritz, Muon anomalous magnetic moment through the leptonic Higgs portal, Phys. Rev. D 95 (2017) 075003 [arXiv: 1606.04943] [INSPIRE].

[7] MuON G-2 collaboration, Final report of the muon E821 anomalous magnetic moment measurement at BNL, Phys. Rev. D 73 (2006) 072003 [hep-ex/0602035] [INSPIRE].

[8] Muon G-2 collaboration, Measurement of the positive muon anomalous magnetic moment to 0.46 ppm, Phys. Rev. Lett. 126 (2021) 141801 [arXiv:2104.03281] [INSPIRE].

[9] T. Aoyama et al., The anomalous magnetic moment of the muon in the Standard Model, Phys. Rept. 887 (2020) 1 [arXiv: 2006. 04822] [InSPIRE].

[10] M.J. Dolan, F. Kahlhoefer, C. McCabe and K. Schmidt-Hoberg, A taste of dark matter: flavour constraints on pseudoscalar mediators, JHEP 03 (2015) 171 [Erratum ibid. 07 (2015) 103] [arXiv: 1412.5174] [INSPIRE].

[11] S. Ipek, D. McKeen and A.E. Nelson, A renormalizable model for the galactic center gamma ray excess from dark matter annihilation, Phys. Rev. D 90 (2014) 055021 [arXiv:1404.3716] [INSPIRE]. 
[12] J.M. No, Looking through the pseudoscalar portal into dark matter: novel mono-Higgs and mono-Z signatures at the LHC, Phys. Rev. D 93 (2016) 031701 [arXiv:1509.01110] [INSPIRE].

[13] N.F. Bell, G. Busoni and I.W. Sanderson, Self-consistent dark matter simplified models with an s-channel scalar mediator, JCAP 03 (2017) 015 [arXiv: 1612.03475] [INSPIRE].

[14] D. Goncalves, P.A.N. Machado and J.M. No, Simplified models for dark matter face their consistent completions, Phys. Rev. D 95 (2017) 055027 [arXiv:1611.04593] [InSPIRE].

[15] M. Bauer, U. Haisch and F. Kahlhoefer, Simplified dark matter models with two Higgs doublets: I. Pseudoscalar mediators, JHEP 05 (2017) 138 [arXiv:1701.07427] [INSPIRE].

[16] N.F. Bell, G. Busoni and I.W. Sanderson, Two Higgs doublet dark matter portal, JCAP 01 (2018) 015 [arXiv:1710.10764] [INSPIRE].

[17] G. Arcadi, M. Lindner, F.S. Queiroz, W. Rodejohann and S. Vogl, Pseudoscalar mediators: a WIMP model at the neutrino floor, JCAP 03 (2018) 042 [arXiv: 1711.02110] [INSPIRE].

[18] N.F. Bell, G. Busoni and I.W. Sanderson, Loop effects in direct detection, JCAP 08 (2018) 017 [Erratum ibid. 01 (2019) E01] [arXiv: 1803.01574] [INSPIRE].

[19] A. Arhrib, R. Benbrik, M. El Kacimi, L. Rahili and S. Semlali, Extended Higgs sector of 2HDM with real singlet facing LHC data, Eur. Phys. J. C 80 (2020) 13 [arXiv:1811.12431] [INSPIRE].

[20] G. Arcadi, 2HDM portal for singlet-doublet dark matter, Eur. Phys. J. C 78 (2018) 864 [arXiv: 1804.04930] [INSPIRE].

[21] T. Abe, M. Fujiwara, J. Hisano and Y. Shoji, Maximum value of the spin-independent cross section in the 2HDM+a, JHEP 01 (2020) 114 [arXiv:1910.09771] [INSPIRE].

[22] G. Arcadi, G. Busoni, T. Hugle and V.T. Tenorth, Comparing $2 H D M+$ scalar and pseudoscalar simplified models at LHC, JHEP 06 (2020) 098 [arXiv:2001.10540] [INSPIRE].

[23] J.M. Butterworth, M. Habedank, P. Pani and A. Vaitkus, A study of collider signatures for two Higgs doublet models with a pseudoscalar mediator to dark matter, SciPost Phys. Core 4 (2021) 003 [arXiv:2009.02220] [INSPIRE].

[24] N. Chen, B. Wang and C.-Y. Yao, The collider tests of a leptophilic scalar for the anomalous magnetic moments, arXiv:2102.05619 [INSPIRE].

[25] BABAR collaboration, Search for a dark leptophilic scalar in $e^{+} e^{-}$collisions, Phys. Rev. Lett. 125 (2020) 181801 [arXiv:2005.01885] [INSPIRE].

[26] J.D. Bjorken et al., Search for neutral metastable penetrating particles produced in the SLAC beam dump, Phys. Rev. D 38 (1988) 3375 [inSPIRE].

[27] M. Davier and H. Nguyen Ngoc, An unambiguous search for a light Higgs boson, Phys. Lett. B 229 (1989) 150 [INSPIRE].

[28] J. Cao, P. Wan, L. Wu and J.M. Yang, Lepton-specific two-Higgs doublet model: experimental constraints and implication on Higgs phenomenology, Phys. Rev. D 80 (2009) 071701 [arXiv:0909.5148] [INSPIRE].

[29] A. Broggio, E.J. Chun, M. Passera, K.M. Patel and S.K. Vempati, Limiting two-Higgs-doublet models, JHEP 11 (2014) 058 [arXiv: 1409.3199] [INSPIRE].

[30] L. Wang and X.-F. Han, A light pseudoscalar of $2 H D M$ confronted with muon $g-2$ and experimental constraints, JHEP 05 (2015) 039 [arXiv:1412.4874] [INSPIRE]. 
[31] T. Abe, R. Sato and K. Yagyu, Lepton-specific two Higgs doublet model as a solution of muon g-2 anomaly, JHEP 07 (2015) 064 [arXiv: 1504.07059] [INSPIRE].

[32] E.J. Chun and J. Kim, Leptonic precision test of leptophilic two-Higgs-doublet model, JHEP 07 (2016) 110 [arXiv: 1605.06298] [INSPIRE].

[33] X.-F. Han, T. Li, L. Wang and Y. Zhang, Simple interpretations of lepton anomalies in the lepton-specific inert two-Higgs-doublet model, Phys. Rev. D 99 (2019) 095034 [arXiv: 1812.02449] [INSPIRE].

[34] L. Wang and Y. Zhang, $\mu$ - $\tau$-philic Higgs doublet model confronted with the muon $g-2, \tau$ decays, and LHC data, Phys. Rev. D 100 (2019) 095005 [arXiv: 1908. 03755] [INSPIRE].

[35] D. Sabatta, A.S. Cornell, A. Goyal, M. Kumar, B. Mellado and X. Ruan, Connecting muon anomalous magnetic moment and multi-lepton anomalies at LHC, Chin. Phys. C 44 (2020) 063103 [arXiv: 1909.03969] [INSPIRE].

[36] S. Jana, V.P.K. and S. Saad, Resolving electron and muon $g-2$ within the $2 H D M$, Phys. Rev. D 101 (2020) 115037 [arXiv:2003.03386] [INSPIRE].

[37] S. Jana, P.K. Vishnu, W. Rodejohann and S. Saad, Dark matter assisted lepton anomalous magnetic moments and neutrino masses, Phys. Rev. D 102 (2020) 075003 [arXiv: 2008.02377] [INSPIRE].

[38] C.-H. Chen, C.-W. Chiang and T. Nomura, Muon g- 2 in two-Higgs-doublet model with type-II seesaw mechanism, arXiv:2104.03275 [INSPIRE].

[39] P. Athron, C. Balázs, D.H. Jacob, W. Kotlarski, D. Stöckinger and H. Stöckinger-Kim, New physics explanations of $a_{\mu}$ in light of the FNAL muon $g-2$ measurement, arXiv:2104.03691 [INSPIRE].

[40] J. Kalinowski and S. Pokorski, Higgs particle production at LEP in multidoublet scenarios with hierarchy of the vacuum expectation values, Phys. Lett. B 219 (1989) 116 [INSPIRE].

[41] J. Kalinowski and M. Krawczyk, Fermion mass effects on $\Gamma(Z \rightarrow b \bar{b}+$ a light Higgs $)$ in a two Higgs doublet model, Phys. Lett. B 361 (1995) 66 [hep-ph/9506291] [INSPIRE].

[42] J. Kalinowski and M. Krawczyk, Two Higgs doublet models and the Yukawa process at LEP-1, Acta Phys. Polon. B 27 (1996) 961 [hep-ph/9602292] [InSPIRE].

[43] S. Dawson and L. Reina, Associated Higgs production in the MSSM, Phys. Rev. D 60 (1999) 015003 [hep-ph/9812488] [INSPIRE].

[44] B. Grzadkowski, J.F. Gunion and J. Kalinowski, Finding the CP-violating Higgs bosons at $e^{+} e^{-}$colliders, Phys. Rev. D 60 (1999) 075011 [hep-ph/9902308] [INSPIRE].

[45] OPAL collaboration, Search for Yukawa production of a light neutral Higgs boson at LEP, Eur. Phys. J. C 23 (2002) 397 [hep-ex/0111010] [INSPIRE].

[46] E.J. Chun and T. Mondal, Searching for a light Higgs boson via the Yukawa process at lepton colliders, Phys. Lett. B 802 (2020) 135190 [arXiv:1909.09515] [INSPIRE].

[47] J.F. Gunion, H.E. Haber, G.L. Kane and S. Dawson, The Higgs hunter's guide, Front. Phys. 80 (2000) 1 [INSPIRE].

[48] A. Djouadi, The anatomy of electro-weak symmetry breaking. II. The Higgs bosons in the minimal supersymmetric model, Phys. Rept. 459 (2008) 1 [hep-ph/0503173] [INSPIRE]. 
[49] G.C. Branco, P.M. Ferreira, L. Lavoura, M.N. Rebelo, M. Sher and J.P. Silva, Theory and phenomenology of two-Higgs-doublet models, Phys. Rept. 516 (2012) 1 [arXiv:1106.0034] [INSPIRE].

[50] J. Alwall, M. Herquet, F. Maltoni, O. Mattelaer and T. Stelzer, MadGraph 5: going beyond, JHEP 06 (2011) 128 [arXiv:1106.0522] [INSPIRE]

[51] J. Alwall et al., The automated computation of tree-level and next-to-leading order differential cross sections, and their matching to parton shower simulations, JHEP 07 (2014) 079 [arXiv: 1405.0301] [INSPIRE].

[52] T. Sjöstrand, S. Mrenna and P.Z. Skands, PYTHIA 6.4 physics and manual, JHEP 05 (2006) 026 [hep-ph/0603175] [INSPIRE].

[53] T. Sjöstrand et al., An introduction to PYTHIA 8.2, Comput. Phys. Commun. 191 (2015) 159 [arXiv: 1410.3012] [INSPIRE].

[54] H. Abramowicz et al., The International Linear Collider technical design report - volume 4: detectors, arXiv:1306.6329 [INSPIRE].

[55] S. Jadach, Z. Was, R. Decker and J.H. Kühn, The tau decay library TAUOLA: version 2.4, Comput. Phys. Commun. 76 (1993) 361 [InSPIRE].

[56] DELPHES 3 collaboration, DELPHES 3, a modular framework for fast simulation of a generic collider experiment, JHEP 02 (2014) 057 [arXiv:1307.6346] [INSPIRE].

[57] S. Catani, Y.L. Dokshitzer, M.H. Seymour and B.R. Webber, Longitudinally invariant $K_{t}$ clustering algorithms for hadron hadron collisions, Nucl. Phys. B 406 (1993) 187 [InSPIRE].

[58] S.D. Ellis and D.E. Soper, Successive combination jet algorithm for hadron collisions, Phys. Rev. D 48 (1993) 3160 [hep-ph/9305266] [INSPIRE].

[59] D. Jeans and G.W. Wilson, Measuring the CP state of tau lepton pairs from Higgs decay at the ILC, Phys. Rev. D 98 (2018) 013007 [arXiv: 1804.01241] [InSPIRE].

[60] A. Hocker et al., TMVA - toolkit for multivariate data analysis, physics/0703039 [INSPIRE].

[61] T. Han, S.K. Kang and J. Sayre, Muon $g-2$ in the aligned two Higgs doublet model, JHEP 02 (2016) 097 [arXiv: 1511.05162] [INSPIRE].

[62] A. Cherchiglia, D. Stöckinger and H. Stöckinger-Kim, Muon g- 2 in the 2HDM: maximum results and detailed phenomenology, Phys. Rev. D 98 (2018) 035001 [arXiv:1711.11567] [INSPIRE].

[63] T. Enomoto and R. Watanabe, Flavor constraints on the two Higgs doublet models of $Z_{2}$ symmetric and aligned types, JHEP 05 (2016) 002 [arXiv: 1511.05066] [INSPIRE].

[64] T. Abe, R. Sato and K. Yagyu, Muon specific two-Higgs-doublet model, JHEP 07 (2017) 012 [arXiv: 1705.01469] [INSPIRE].

[65] Y. Omura, E. Senaha and K. Tobe, Lepton-flavor-violating Higgs decay $h \rightarrow \mu \tau$ and muon anomalous magnetic moment in a general two Higgs doublet model, JHEP 05 (2015) 028 [arXiv: 1502.07824] [INSPIRE]. 